\title{
Multimodal Preparation Package: Improving Patients' Awareness and Satisfaction with Day Case Cataract Surgery
}

\author{
Basma M. Khalil (1), (2) Sabah N. Elderiny, (3) Wafaa Kh Ibrahem, (4) Shimaa f. Miky and \\ (5) Mohamed A. Rashad \\ Departments of Medical - Surgical Nursing and 3,4Community Health Nursing, Faculty of Nursing, \\ 5Ophthalmic Surgery, Faculty of Medicine, Ain Shams and 2Helwan Universities
}

\begin{abstract}
Aim: This study aimed to evaluate the effect of multimodal preparation package on improving patients`awareness and satisfaction with day case cataract surgery. Subjects and Method: A quasi-experimental design was utilized for the conduction of this study in the Ophthalmic Outpatients`Clinics and Surgical Waiting Room of the Ophthalmic Unit, at Ain Shams University and Specialized Hospitals. A purposive sample composed of 60 patients undergoing cataract surgery, adults and old age, from both genders and were recruited from the above mentioned settings. Tools: 1) Patients' interviewing questionnaire (pre / post tests), to determine the knowledge regarding day case cataract surgery, 2) An observation checklist (pre / post tests), to evaluate studied patients' practices in relation to day case cataract surgery (pre / post tests), 3) Patients' anxiety concerns pre surgery (pre test), 4) Patients` satisfaction assessment sheet (post test), to assess the satisfaction level and 5) Patients` health condition assessment sheet (post and follow up tests). Results: More than two fifths of the studied patients were females, married and smoke. Moreover, they had anxiety concerns pre surgery added to their awareness level (knowledge and practices) were unsatisfactory in pre test. Conclusion: The current study concluded that, multimodal preparation package had a positive effect on improving awareness (knowledge and practices) and satisfaction among patients with day case cataract surgery, whereas significant improvement was found in post assessment. In addition, patients` health condition assessment was satisfactory in post and follow - up tests, added to its positive relation with their awareness. Recommendations: Further studies should be carried out on a large number of patients with day case cataract surgery for evidence of results and generalization.
\end{abstract}

Key words: Day case cataract surgery - Awareness - Satisfaction - Multimodal preparation package Introduction

Cataract is an opacity of the eye lens which can lead to blurry vision or blindness whereas with age it grows progressively to be darker and more dense preventing light from easily passing through the lens and contributing to low quality of life. It may be classified into three categories: age-related cataracts, metabolic cataracts, and cataracts secondary to other causes. Age-related cataracts are the most common type in adults (Koolwijk et al., 2015 \& Liu et al., 2017).
Patients with cataracts have difficulty seeing in poorly lit environments. Many people also experience increase sensitivity to light and glare, double vision (or ghost images) and fading colors (blue may appear green and white may appear dull beige). The risk factors for cataract development include gender, diabetes mellitus, exposure to excessive sunlight, life style, eye injury and use of steroids (Lin et al., 2013 \& Wei et al., 2016). 
Day surgery is fast, safe therapeutic regimen whereas the surgery and discharge occur within $24 \mathrm{hrs}$. It is becoming increasingly common throughout the world. A combination of new developments in surgical technique and technology, changes in hospital resources allocations and patient demands for quicker, more effective treatments have placed day surgery at the forefront of modern patient management (Casparis et al., 2012 \& Zhuang et al., 2018). Day surgery of cataract is more costeffective and efficient than inpatient surgery with equivalent clinical outcomes. It provides patients with familiar environment on their own home, reduces the waiting list time, enhances visual rehabilitation, favoring for the doctor and the patient alike (Agarwal \& Kumar, 2011, Hamed \& Fedorowicz, 2011 and Zhao et al., 2015)

Cataract surgery include: Intra capsular cataract extraction whereas the entire lens capsule was removed. Extra capsular cataract extraction in which the lens capsule is incised and the lens cortex and nucleus are removed leaving the posterior capsule and lens zonule in place. Small incision cataract surgery which results in a good visual outcome. Lastly Phacoemulsification is a process of the lens nucleus fragment ion with ultrasonic vibrations and aspirating the lens material through a double - lumen, irrigationaspiration system that requires only a small incision (Lundstrom et al., 2011 \& Rengaraj et al., 2012). Moreover in cataract surgery, the eye lens was removed and replaced with an artificial lens (intraocular lens) which is a clear plastic lens that becomes a permanent part of the eye, it cannot be felt. The surgery requires much less time. The incision in the eye will take 4 weeks to completely heal, during that time the vision will gradually improve. It can be performed either under local twilight or general anesthesia (Bucher et al., 2014).
Many other remedies for cataracts have been attempted included medications, eye drops, vitamins, changes in diet and eyes rest. Early complications include: corneal edema raised intraocular pressure, corneal abrasion, wound leak, suture complications, iris prolapse, incarcerated vitreous, severe anterior uveitis and displacement of the intraocular lens. Later complications include: Cystoids' macular edema, endophthalmitis, retinal detachment, posterior capsule opacification and unsatisfactory refractive error (Hinkle \& Cheever,2014).

Several studies have shown that patients` education and appropriate preparation would positively affect their health promotion, knowledge, attitudes, skills and behaviors. It has also been shown that education would reduce their anxiety, increase adaptability pre surgery and could decrease the length of hospital stay (Moradi \& Hajbaghery, 2015). A comprehensive multimodal preparation package consisting of individualized instructions, providing an educational pamphlet, showing an educational film and performing an orientation round in the Ophthalmology department could be effective in improving patients' condition (Hajbaghery et al., 2014 \& Elsaay et al., 2016).

Satisfaction of patients with cataract surgery referred to an expression of overall judgment on care quality particularly in the interpersonal process aspect. It was defined as patients` opinions of care received from staff of nurses during hospitalization period. Patients ' satisfaction with medical treatment and nursing management has been reported as the most important predictor of the overall satisfaction with hospital care and an important goal of any health care organization (Kleefstra et al., 2012 \& Nettina, 2014). 
Significance of the study:

Today, cataract is a greater problem and day case surgery includes concepts of care other than immediate discharge of patient after initial recovery from anesthesia. In the United States, more than 2.5 million people have cataract surgery each year and age related cataract which represents about 18 million people is responsible for $48 \%$ of world blindness. Women had a higher prevalence of cataract (Taha, \& AbdElaziz, 2015 and Dewit et al., 2016). In Egypt, the prevalence of low vision for all ages is $47.9 \%$ of the population aged $65 \mathrm{yrs}$ and major causes for blindness are cataract $(54.8 \%)$. Patients may have been discharged from the hospital inadequately prepared to perform their own eye care post surgery. So, there is a need to initiate, update and reinforce patients' teaching to affects on their experiences and recovery (Hegazy et al., 2012 \& El-Shamy et al., 2017)

\section{Aim of the study}

This study aimed to evaluate the effect of multimodal preparation package on improving patients awareness and satisfaction with day case cataract surgery. This aim was achieved as follows:

- Assess patients' knowledge and practices as regards day case cataract surgery

- Identify patients`satisfaction level with day case cataract surgery

Develop and implement the multimodal preparation package for the studied patients.

- Evaluate its effect on their knowledge and practices, added to satisfaction level.

\section{Hypothesis:}

It was hypothesized that, the multimodal preparation package had a positive effect on awareness and satisfaction among patients having day case cataract surgery.

\section{Subjects and methods}

\section{Operational definitions:}

Awareness: means patients knowledge and practices

Day case surgery: means performance of a surgical procedure that occurs without overnight admission of the patient prior to or following the intervention.

With: means pre / post surgery and follow - up period.

Multimodal preparation package: means individualized face-to-face patient education, provision of an educational pamphlet, presenting a video, and performing a round in the Ophthalmologic department

\section{Research design:}

A quasi-experimental design was utilized to conduct this study

\section{Setting:}

The study was carried out in the Ophthalmologic Outpatients`Clinics and Surgical waiting room of the Ophthalmologic Unit at Ain Shams University Hospital and Ain Shams Specialized Hospital.

\section{Subjects:}

A purposive sample was composed of 160 cataract patients, adults and old age, from both genders. They were recruited from the above mentioned settings as follows: 
- Patients were taken from Ain Shams University Hospital $(\mathrm{n}=30)$.

Patients were taken from Ain Shams Specialized Hospital $(n=30)$.

\section{Inclusion criteria:}

- Conscious adult patients with cataract surgery for the first time.

- $\quad$ Patients are willing to participate in the study

- $\quad$ Patients with the same protocol of local anesthesia and management

- Patients without ocular co - morbid conditions

\section{Tools of data collection:}

1- Patients' interviewing questionnaire (pre / post and follow up tests). It was designed by the researchers after reviewing the related literature and consulting the experts to assess studied patients' knowledge about day case cataract surgery. It was written in simple Arabic language and divided into the following parts:

- Characteristics of the studied patients such as: age, gender, marital status, income, educational level and smoking.

- Patients' medical records to identify past, present medical and surgical history, diagnosis, diagnostic measures and management.

- Patients` knowledge assessment sheet: It included cataract definition, causes, characteristics, diagnosis and management, cataract surgery, Preoperative preparations such as physical and psychological preparations, postoperative care such as pain relieve, medications, correct position, diet and discharge instructions (daily activities, follow - up visit, immediate calling, treatment and permissible activities).

\section{Scoring system:}

Patients' answers were scored as (1) for correct answer and (zero) for incorrect answer. The total score was sorted into either satisfactory level (70\% and more) or unsatisfactory level (less than 70\%).

\section{II- An observation checklist (pre /} post and follow up tests). It adapted from Lewis et al. (2014) \& Nettina et al. (2014) and Dewit et al. (2016). It was developed and filled by the researchers to evaluate studied patients' practices in relation to day case cataract surgery: pain management, breathing and legs exercises, correct position, eye care, hygienic measures, ambulation, eye drop instillation and ointment application.

\section{1- Scoring system:}

A correct practice was scored as (1), while the incorrect (zero). It was scored into either inadequately done (less than $70 \%$ ) or adequately done (70\% and more).The total score was categorized as satisfactory $=70-$ 100 , or unsatisfactory $=$ less than 70 .

III- Patients' anxiety concerns pre surgery (pre test). It was developed by the researchers in light of the relevant and related literatures to evaluate patients concerns as regards: Anesthesia, pain, physical injuries isolation, prognosis, deformity and loss of self control.

\section{IV- Patients` satisfaction assessment} sheet (post test). It was based on Kleefstra et al. (2012) and composed of a core questionnaire for the assessment of patient satisfaction for general day care (COPS-D). It was consisted of six dimensions: Admission procedure, nursing care, medical care, 
information, autonomy, discharge and aftercare.

The COPS-D contain 17 questions. The answer was sorted by a 5 - point Likertscale $(1=$ unsatisfied, $2=$ somewhat satisfied, $3=$ rather satisfied, $4=$ quite satisfied and $5=$ very satisfied). scale $=85$

Total $=17$ questions $\times 5$ point Likert-

High satisfaction (51 - 85) and

Low satisfaction (Less than 51).

V- Patients health condition assessment sheet: (post and follow up tests). It was developed by the researchers in light of the relevant and related literatures to evaluate patients 'health conditions on $1^{\text {st }}$ follow up visit to Out patients' Clinics after one week post surgery and after one month later. to determine prognosis of patients' surgery through the presence or absence of the following: sudden severe pain, hallos around lights, redness of the eye lid, increase eye discharge, eye drainage to greenish color, blurring vision, diplopia, persistent headache and wound adherence.

\section{Scoring system:}

- Each item was scored as "0" for no and "1" for yes. The total score was categorized as satisfactory $=50-100 \%$, or unsatisfactory $=$ less than $50 \%$.

\section{Content validity:}

It was ascertained by a group of experts from Ophthalmic Surgery, MedicalSurgical Nursing and Community Health Nursing. Their opinions were elicited regarding to the tools format layout, consistency and scoring system. Contents of the tools were tested regarding to the knowledge accuracy, relevance and competence.
Ethical considerations:

In the planning stage approval was obtained from the directors of the above mentioned settings. All patients were informed about the study and their rights according to medical research ethics that they were free to decide whether or not they would participate in the study. Then a written informed consent was obtained from each patient who agreed to participate in the study.

\section{Pilot study:}

A pilot trial was carried out on $10 \%$ of the total sample to test practicability and clarity of study tools, added to sample and settings. Pilot sample was later involved in the study as there were no radical modifications in the study tools.

\section{Procedures:}

- Sampling was started and completed within 6 months.

o Purpose of the study was explained to the patients who agreed to participate in the study prior to any data collection.

o The researchers started to collect the data from the studied patients using the pre constructed tools as follows:

- On the same day of diagnosis when patients came to Ophthalmologic Outpatients`Clinics and Surgical waiting room of the Ophthalmologic Unit

- On the first follow - up visits when the patients came to Ophthalmologic Outpatients`Clinics

o Filling in the tools was done by the researchers according to the patients' understanding and health condition. 
o The data were collected by the researchers 3 days/ week at the morning and afternoon shifts of the surgical time.

o The preparation package was designed based on analysis of the actual patients' needs assessment by using the pre constructed tools. Content of the multimodal preparation package was consistent with the related literatures (national and international), presented with theoretical and practical sessions.

o Patients were divided into small groups including $4-5$ and each group obtained 4 sessions ( 2 theory and 2 practice). At first simple written instructions were distributed and orientation about the objective and outline was done.

○ The theoretical part was implemented through lectures and group discussions, using data show and poster as a media. It was taken in 2 sessions (each session for 45 minutes). During this session, cataract surgery was explained to them. Also, the patients were provided by an educational pamphlet with some explanations about cataract surgery: patients' assessment, care pre- surgery, morning care of the surgery, immediately care post surgery, discharge instructions, home care and follow - up visits. Then, pictures of different parts of the Ophthalmology Unit and a 10-minute video describing the environment of the unit, the process of cataract surgery, pre- and postsurgery care, and experiences of a patient who had undergone the same surgery was shown to the patients. Finally, the researcher answered their questions

○ The practical part was implemented through demonstration, re- demonstration and video. It was taken in 2 sessions (each session for one hour) and covers the following items: The first session was related to infection control, eye drop instillation and ointment application. Second session delt with correct position, permissible activities, breathing and leg exercises.

o Patients were informed to be in contact with the researchers by telephone for any guidance.

o Patients were assessed either individually or in groups that entail 4-5

$\circ$ Evaluating the preparation package effect on the studied patients as regards:

- Knowledge and practices by using post - test (immediately after multimodal preparation package sessions) and follow- up test ( 2 months later) by the same tools.

- Anxiety concerns pre surgery

- Levels of satisfaction using post test

- Health condition assessment sheet (unusual complaints) using post and follow up tests by the same tools.

\section{Results:}

Table (1): Presents characteristics of the studied patients. Results revealed that mean age of them was $43.5 \pm 11.6$ and more than half of them 60.0 were working. As regards to gender, marital status and smoking more than two fifths of them were females, married and smoke (53.3, 51.7 \& 45.0 respectively). Concerning education, one third (33.3) of them were with low education. In relation to job, more than one third of them had sedentary work $(30 \%$ \& $32.5 \%$ respectively).

Table (2): Reveals studied patients anxiety concerns pre cataracts surgery. As noticed, more than three quarters of them were fears from anesthesia and pain (80.0 
\&76.7 respectively). Meanwhile, nearly one third of them were fear from deformity and loss of self control $\quad(35.0 \quad \& \quad 31.7$ respectively).

Table (3): Shows studied patients satisfactory knowledge about cataract surgery care pre/post preparation package. Results indicated significant improvement in patients' knowledge regarding post and follow - up assessment (mean percent $=48.0$ $\pm 2.5 \& 54.2 \pm 2.7$ respectively) compared to pre - assessment $(15.9 \pm 3.4)$, with $\mathrm{t}-$ test $=$ 59.4 and 12.4 respectively), $p<0.05$.

Table (4): Reveals studied patients satisfactory practices regarding cataract surgery care pre/post preparation package. Results showed significant improvement in patients' practices regarding post and follow - up assessment (mean percent $=44.1 \pm 3.9$ $\& 54.1 \pm 1.2$ respectively) compared to pre - assessment $(12.4 \pm 2.7)$, with $\mathrm{t}-$ test $=52.8$ $\& 5.4$ respectively), $p<0.05$.

Table (5): Presents studied patients satisfaction level post cataract surgery. As observed more than three fourths $77.6 \%$ of them were satisfied for the following: Preadmission period, admission preparations, operative room, nursing management, medical treatment, information, autonomy and discharge instructions, with mean number $=46.6 \pm 2.3$ and percent of mean $=$ $77.6 \%$.

Table (6): Presents unusual complaints among studied patients post cataract surgery. There is a highly significant difference between post and follow-up assessment regarding to patients complaints $(\mathrm{t}=21.9, \quad \mathrm{P}<0.001)$, whereas significant improvement was indicated in follow-up assessment compared to post assessment (2.8 $\pm 0.7 \& 7.4 \pm 1.5$ respectively)

\section{Table (1): Characteristics of the studied patients $(n=60)$}

\begin{tabular}{|l|c|c|}
\hline \multicolumn{1}{|c|}{ Items } & No & \% \\
\hline Age / years $(\overline{\mathbf{X}} \pm$ SD) & \multicolumn{2}{|c|}{$43.5 \pm 11.6$} \\
\hline Gender & 28 & 46.7 \\
Male & 32 & 53.3 \\
$\quad$ Female & 31 & \\
\hline Marital status & 29 & 51.7 \\
$\quad$ Married & & 48.3 \\
$\quad$ Unmarried & 22 & 36.7 \\
\hline Level of education & 18 & 30.0 \\
$\quad$ High & 20 & 33.3 \\
Moderate & 24 & 40.0 \\
$\quad$ Low & 36 & 60.0 \\
\hline Job & & \\
Not working & 27 & 45.0 \\
$\quad$ Working & 33 & 55.0 \\
\hline Smoking & & \\
Present & & \\
Absent & & \\
\hline
\end{tabular}


Multimodal Preparation Package: Improving Patients' Awareness and Satisfaction with Day Case Cataract Surgery

Table (2): Presentation of anxiety concerns among studied patients pre cataract surgery

\begin{tabular}{|l|c|c|}
\hline \multicolumn{1}{c|}{ Anxiety concerns } & \multicolumn{2}{c|}{ Studied patients (n=60) } \\
\cline { 2 - 3 } & \multicolumn{1}{c|}{ No } & \% \\
\hline Anesthesia & 48 & 80.0 \\
\hline Pain & 46 & 76.7 \\
\hline Physical injuries & 38 & 63.3 \\
\hline Isolation & 40 & 66.7 \\
\hline Prognosis & 42 & 70.0 \\
\hline Deformity & 21 & 35.0 \\
\hline Loss of self control & \multicolumn{2}{|c|}{$\mathbf{3 6 . 3 \pm \mathbf { 1 1 . 6 }}$} \\
\hline \multicolumn{2}{|c|}{$\mathbf{6 0 . 5 \%}$} \\
\hline
\end{tabular}

Table (3): Presentation of studied patients` satisfactory knowledge regarding cataract surgery care pre/post preparation package

\begin{tabular}{|l|c|c|c|}
\hline \multirow{2}{*}{\multicolumn{1}{|c|}{ Items }} & \multicolumn{2}{c|}{ Studied patients (n=60) } \\
\cline { 2 - 4 } & Pre & Post & Follow- up \\
\hline Information about cataract surgery & $15(25.0)$ & $50(83.3)$ & $57(95.0)$ \\
\hline Physical preparation - & $8(13.3)$ & $46(76.7)$ & $56(93.3)$ \\
\hline Psychological preparations & $17(28.3)$ & $48(80.0)$ & $54(90.0)$ \\
\hline Infection prevention & $22(36.7)$ & $47(78.3)$ & $51(85.0)$ \\
\hline Pain / anxiety reduction & $19(31.7)$ & $49(81.7)$ & $55(91.7)$ \\
\hline Positioning & $15(25.0)$ & $52(86.7)$ & $57(95.0)$ \\
\hline Safety measures & $16(26.7)$ & $46(76.7)$ & $54(90.0)$ \\
\hline Permissible activities of daily living & $14(23.3)$ & $48(80.0)$ & $56(93.3)$ \\
\hline Nutrition & $13(21.7)$ & $44(73.3)$ & $51(85.0)$ \\
\hline Medications and eye care & $17(28.3)$ & $49(81.7)$ & $57(95.0)$ \\
\hline Exercises technique & $14(23.3)$ & $52(86.7)$ & $55(91.7)$ \\
\hline Sexual activity & $16(26.7)$ & $48(80.0)$ & $56(93.3)$ \\
\hline Transporting / traveling & $13(21.7)$ & $46(76.7)$ & $50(83.3)$ \\
\hline Social support & $22(36.7)$ & $47(78.3)$ & $49(81.7)$ \\
\hline Communication with health care team & $19(31.7)$ & $44(73.3)$ & $51(85.0)$ \\
\hline Complications prevention & $15(25.0)$ & $49(81.7)$ & $55(91.7)$ \\
\hline Discharge instructions & $16(26.7)$ & $52(86.7)$ & $57(95.0)$ \\
\hline $\bar{X}$ No \pm SD & $\mathbf{1 5 . 9} \mathbf{3 . 4}$ & $\mathbf{4 8 . 0 \pm 2 . 5}$ & $\mathbf{5 4 . 2} \pm \mathbf{2 . 7}$ \\
\hline T - value & $\mathbf{T 1}$ between pre \& post tests = 59.4* \\
\cline { 2 - 4 } & $\mathbf{T 2 ~ b e t w e e n ~ p o s t ~ \& ~ f o l l o w - ~ u p ~ t e s t s ~ = ~ 1 2 . 4 * ~}$ \\
\hline
\end{tabular}


Table (4): Presentation of studied patients` satisfactory practices regarding cataract surgery care pre/post preparation package

\begin{tabular}{|l|c|c|c|}
\hline \multirow{2}{*}{\multicolumn{1}{|c|}{ Items }} & \multicolumn{2}{c|}{ Studied patients (n=60) } \\
\cline { 2 - 4 } & Pre & Post & Follow- up \\
\hline - Positioning technique & $13(21.7)$ & $46(76.7)$ & $55(91.7)$ \\
\hline - Eye care & $12(20.0)$ & $44(73.3)$ & $53(88.3)$ \\
\hline - Eye irrigation & $9(15.0)$ & $36(60.0)$ & $54(90.0)$ \\
\hline - Infection control measures & $16(26.7)$ & $45(75.0)$ & $53(88.3)$ \\
\hline - Eye drop instillation & $15(25.0)$ & $49(81.7)$ & $56(93.3)$ \\
\hline -Ointment application & $13(21.7)$ & $44(73.3)$ & $55(91.7)$ \\
\hline - Breathing and legs exercises & $9(15.0)$ & $45(75.0)$ & $53(88.3)$ \\
\hline $\bar{X}$ No \pm SD & $\mathbf{1 2 . 4} \pm \mathbf{2 . 7}$ & $\mathbf{4 4 . 1} \pm \mathbf{3 . 9}$ & $\mathbf{5 4 . 1} \pm \mathbf{1 . 2}$ \\
\hline \multirow{2}{*}{ T - value } & T1 between pre \& post tests = 52.8* \\
\cline { 2 - 4 } & T2 between post \& follow- up tests = 5.4* \\
\hline
\end{tabular}

Table (5): Presentation of studied patients`satisfaction level post cataract surgery

\begin{tabular}{|l|c|c|}
\hline \multicolumn{1}{|c|}{ Items } & \multicolumn{2}{c|}{ High patients`satisfaction (n=60) } \\
\cline { 2 - 3 } & No & \% \\
\hline Pre-admission period & 46 & 76.7 \\
\hline Admission preparation & 44 & 73.3 \\
\hline Operative room & 47 & 78.3 \\
\hline Nursing management & 46 & 85.0 \\
\hline Medical treatment & 51 & 78.3 \\
\hline Information & 47 & 73.3 \\
\hline Autonomy & 44 & 80.0 \\
\hline Discharge instructions & 48 & \multicolumn{2}{|c|}{$\mathbf{4 6 . 6 \pm \mathbf { 2 . 3 }}$} \\
\hline \multicolumn{2}{|c|}{$\mathbf{7 7 . 6 \%}$} \\
\hline
\end{tabular}


Multimodal Preparation Package: Improving Patients' Awareness and Satisfaction with Day Case Cataract Surgery

Table (6): Presentation of unusual complaints among studied patients post cataract surgery

\begin{tabular}{|c|c|c|c|c|}
\hline \multirow{3}{*}{ Unusual complaints } & \multicolumn{2}{|c|}{ Post-test } & \multicolumn{2}{|c|}{ Follow-up test } \\
\hline & \multicolumn{2}{|c|}{$\mathrm{n}=60$} & \multicolumn{2}{|c|}{$\mathrm{n}=60$} \\
\hline & No & $\%$ & No & $\%$ \\
\hline Redness of eye lid & 8 & 13.3 & 3 & 5.0 \\
\hline Increase eye discharge & 10 & 16.7 & 4 & 6.7 \\
\hline Change eye drainage & 6 & 10.0 & 3 & 5.0 \\
\hline Sudden severe pain & 9 & 15.0 & - & - \\
\hline Blurring vision, diplopia & 6 & 10.0 & 2 & 3.3 \\
\hline Persistent headache & 7 & 11.7 & 2 & 3.3 \\
\hline Wound adherence & 6 & 10.0 & - & - \\
\hline Hallos around light & 7 & 11.7 & 3 & 5.0 \\
\hline$\overline{\mathrm{X}}$ No $\pm \mathrm{SD}$ & \multicolumn{2}{|c|}{$7.4 \pm 1.5$} & \multicolumn{2}{|c|}{$2.8 \pm 0.7$} \\
\hline \% of Mean & \multicolumn{2}{|c|}{$12.3 \%$} & \multicolumn{2}{|c|}{$4.7 \%$} \\
\hline $\mathbf{T}$ - value & \multicolumn{4}{|c|}{$T$ test between post $\&$ follow- up tests $=21.9 *$} \\
\hline
\end{tabular}

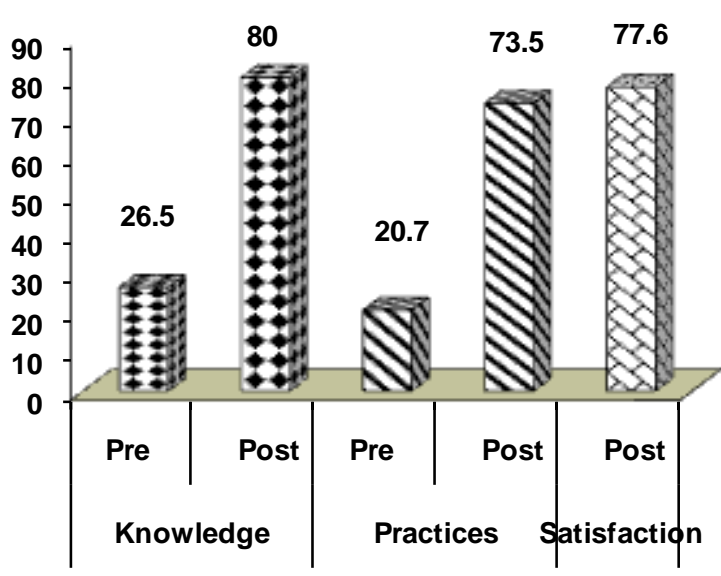

Fig. (1): Presentation of studied patients` awareness and satisfaction levels

Discussion

Cataract is considered from the major causes of severe impairment. Education has a vital role in improving knowledge and practices of the patients and consequently improve the quality of life. Teaching patients is very important, however, it is widely advocated but frequently neglected (Casparis et al., 2012 \& Zhuang et al., 2018). The current study aimed to evaluate the effect of multi-modal preparation package on improving patients` awareness and satisfaction with day case cataract surgery.

In the present study, findings regarding to patients' characteristics revealed that, mean 
age of them was $43.5 \pm 11.6$. This finding was supported by Koolwijk et al.(2015) \& El-Shamy et al.(2017) who stated that cataract is the most common cause of poor vision in the elderly and as many as $85 \%$ will have some lens clouding by the time they reach 80 years of age. Lundstrom et al. (2011) claimed that cataract is one of the few normal physiological changes in aging process.

Considering studied patients`anxiety concerns pre cataracts surgery. As noticed, more than half of them were fears from: Anaesthesia, pain, physical injuries, isolation, prognosis, deformity and loss of self-control. Hegazy et al.((2008) \& Dewit et al. (2016) discussed that, anxiety activates sympathetic nervous system which characterized by an increase in catecholamine concentration, heart rate, blood pressure and glucocorticoid levels. It also affects immune responses, therefore, there is a need for nursing intervention to reduce anxiety of patients under local anesthesia. Hinkle \& Cheever (2014) and Wei et al. (2016) mentioned that teaching patients represent an important role in the treatment and contributes to decrease anxiety level which results when patients are unable fully to comprehend the world around as regards the surgery.

$\begin{array}{ccc}\text { Concerning studied } & \text { patients' } \\ \text { satisfactory knowledge about cataract }\end{array}$ surgery, results revealed significant improvement post assessment on the following items: medications, psychological / physical preparations, daily living activities to be independent, complications and discharge instructions. The previous findings may be due to lack of patients` information.

Bucher et al. (2014) reported that teaching patients involves dispelling misconceptions and provide correct knowledge about: medications morning of the surgery, wear short-sleeved button front shirt or blouse and no undershirts, pantyhose or girdles, remove all jewellery, do not use scented products (perfume, aftershave, powder, spray) and eye or face make-up.
In the same line, Lewis et al. (2014) \& Dewit et al. (2016) stated that patients should have discharge instructions about: using eye drops and preventing eye injury at least for 12 weeks. Washing eyelid gently with a cotton makeup pad by warm water. Wearing eye shield on bath or hair wash for one week. Gradually increasing daily physical activities. Sleep on back or un operated side for 1 week. Don't rub or bump, use soap or shampoo, bend the head down below waist, get constipated, swim, golf, or play contact sports, drive, strain and lift anything heavier until permitted. Moreover, contact the surgeon if: operated eye becomes very painful or swollen, more mucous discharge and vision reduction.

In addition, Hamed \& Fedorowicz (2011) and Zhao et al. (2015) highlighted that majority of cataract patients needs for pre surgery preparations written and verbal instructions pre discharge. In day surgery because patient's journey is a short one, therefore, they should be will educated and take post discharge advise. In the same line, studied patients' knowledge about psychological redness were increased in post test. Nettina (2014) \& Liu et al. (2017) stated that patients psychological preparations play a vital role in the successful outcome of cataract surgery, so they should be informed about changes after the surgery, effect of psychological factors on the surgery and awareness about physical environment. Moreover, patients learned to attend postoperatively for examination within a few days depending on the treatment protocol and later follow-up examination is usually at one to three weeks..

As regards patient's satisfactory practices, significant improvement was observed in post-test for eye drop instillation, ointment application, infection control, correct position, breathing and leg exercises. These findings may be due to shortage time of hospital stay and poor knowledge about correct practices. According to Lewis et al. (2014) day case surgery should provide a 
major effective form of care with a high quality of services.

Considering unusual complaints among studied patients post cataract surgery. There is a highly significant difference between post and follow-up assessment whereas the progress was obvious in follow - up. The previous finding may be explained as there was no compliance of those patients with the instructions from the first time which result in complications. Hegazy et al. (2012) \& ElShamy et al. (2017) recognized that patients should be informed that at 4 weeks the following complaints should be absent: pain, photophobia, redness (except remaining sub - conjunctival hemorrhage) and cells in the anterior chamber. All mobility permissive patients should be taught to take extreme care of the eyes at home. Taha \& AbdElaziz (2015) stressed that postoperative examination are crucial, so it is customary that the patients be seen the next day, however writing detailed note about what is normal and what complications on the first day is unacceptable. It is imperative that a 24 hour contact number be provided to all patients. Nettina (2014) \& Elsaay et al. (2016) mentioned that patients should be educated about the risks and benefits of the surgery and alternatives to the treatment. Moreover, determine if the expected improvement of the disability outweighs the potential risk, cost and inconvenience of the surgery.

\section{Conclusion}

On light of the current study results, it can be concluded that multimodal preparation package had a positive effect on improving awareness (knowledge and practices) and satisfaction among studied patients with day case cataract surgery whereas significant improvement was found in post assessment. Moreover, patients` health condition assessment was satisfactory in post and follow - up tests added to its positive relation with their awareness.

\section{Recommendations}

Educational sessions should be prepared for patients undergoing cataract surgery.

Patients are in need to a simplified illustrated and comprehensive Arabic booklet including information about cataract surgery.

Further studies should be carried out on a large number of patients with day case cataract surgery for evidence of results and generalization.

\section{References}

Agarwal, A. \& Kumar, D. (2011): Costeffectiveness of cataract surgery. Curr Opin Ophthalmol. 22(1):15-18.

Bucher, L., Dirksen, S., Heitkemper, M. \& Lewis, S. (2014): Medical-Sugical Nursing Assessment and Managment of Clinical Problems, $\left(9^{\text {th }}\right.$ ed.), USA: Mosby, 316-60.

Casparis, H., Lindsley, K., Kuo, I., Sikder, S. \& Bressler, N. (2012): Cataract surgery in people with age-related macular degeneration. The Cochrane Collaboration., 11 (6): 35 - 9.

Dewit, S., Stromberg, H. \& Dallred, C. (2016): Medical-surgical nursing: concepts and practice. Elsevier Health Sciences: 694-7.

Elsaay O., Elshemy M. \& Elsays H. (2016): Effect of a Multi-Modal Preparation Package on knowledge and anxiety among Patients Undergoing cardiac Catheterization. International Journal of Nursing Didactics, 16 (132): 1-12. 
El-Shamy, k., Shabaan,A., Fathy, S. \& ElBanna, M. (2017): Needs Assessment Of Patients Undergoing Cataract Surgery At Mansoura University Ophthalmic Center, IOSR Journal of Nursing and Health Science, Nov-Dec, 6 (10): 1-11.

Hajbaghery M., Moradi T. \& Mohseni R. (2014): Effects of a multimodal preparation package on vital signs of patients waiting for coronary angiography, Nurs Midwifery Study, Apr; 3(1): 17-51.

Hamed W. \& Fedorowicz Z.(2011): Day care versus in-patient surgery for agerelated cataract. Cochrane Database Syst Rev. 1:CD004242.

Hegazy S., Sobeh H., Mohamed H.\& Ahmed N. (2008): Day case cataract surgery: Improving patients self - care practices. The New Egyptian Journal of Medicine. Supplement, 38 (6): 18 - 26.

Hegazy S., Ragheb M., Elsayed N. \& Rashad M. (2012): Health Needs Managements Among Patients Undergoing Day Case Cataract Surgery: Proposed protocol,life science Journal; 9(2): 1316-27.

Hinkle, J. \& Cheever, K.(2014): Textbook of Medical-Surgical Nursing, $\left(13^{\text {th }}\right.$ ed.), Philadelphia: wolters kluwer health, 1389-400.

Kleefstra, S., Kool, R., Zandbelt, L. \& De Haes. J. (2012): An instrument assessing patient satisfaction with day care in hospitals, Research article, May.

Koolwijk J., Fick M., Selles C., Turgut G., Noordergraaf J.,Tukkers F. \& Noordergraaf G. (2015): Outpatient cataract surgery: incident and procedural risk analysis do not support current clinical ophthalmology guidelines.Ophthalmology, 122(2):281-7.
Lewis, L., Dirksen, R., Kemper, M. \& Bucher, L. (2014): Medical-surgical nursing: Assessment and management of clinical problems $\left(9^{\text {th }}\right.$ ed.) Missouri: Mosby, 1037- 38.

Lin J, Fang X. \& Wu S. (2013): The management pattern carried out in a cataract surgery day ward. Eye Sci., 28(2):79-83.

Liu Y., Wilkins M., Kim T, Malyugin B \& Mehta J. (2017): Cataracts. Lancet. 390(10094):600-12.

Lundstrom M., Behndig A., Kugelberg M., Montan P., Stenevi U. \& Thorburn W. (2011): Decreasing rate of capsule complication in cataract surgery, eightyear study of incidence, risk factors and data validity by the Swedish National Cataract Register. J Cataract Refract Surg, 37:1762-67.

Moradi T. \& Hajbaghery M. (2015): The effect of a multi-modal preparation package on anxiety in patients undergoing coronary angiography, Int Cardiovasc Res J., 9(1):10-16.

Nettina, S. (2014): Lippincott Manual of Nursing Practice, $\left(10^{\text {th }}\right.$ ed.), Philadelphia: Wolters Kluwer health, 729-33.

Rengaraj V., David F., Radhakrishnan M., Kenia H., Pariskshit G. \& Sabyasachi S. (2012): Manual Small Incision Cataract Surgery. Asia-Pacific Journal of Ophthalmology, 1(2): 113-19.

Taha,N. \& AbdElaziz, N. (2015): Effect of nursing intervention guidelines on nurses' role, patients' needs and visual problems post cataract surgery. American Journal of Nursing Science, 4 (5): 261-9.

Vincent J. \& Patalano I. (2012): The Risks and Benefits of Cataract Surgery. Digital Journal Of Ophthalmology, 3 (7): 23- 5. 
Wei Y, Liang V, Wu F \& Chen M. (2016): Application of clinical pathway with daycare unit mode among patients with cataract. Chinese Health Quality Management. 23(4):55-7.

Zhao R., Yang L., Zhang W., Liu G., Du N., Liu J., Jia T. \& Zhang W.(2015): Assessment of the development of ambulatory surgery in shanghai municipal hospitals. Chinese Hospitals. 2015;4:710.

Zhuang M., Cao J., Cui M., Yuan S., Liu Q. \& Fan W. (2018): Evaluation of day care versus inpatient cataract surgery performed at a Jiangsu public Tertiary A hospital, BMC Ophthalmology, 18(6): 134. 